\title{
Densifying the Optical Reference Frame: The Tycho-2 Catalog of 2.5 Million Stars
}

\author{
S.E. Urban and G.L. Wycoff \\ U.S. Naval Observatory, Washington DC
}

\begin{abstract}
Since the establishment of the Hipparcos Catalog as the defining source of the optical reference frame, densification beyond its $\approx 120,000$ stars has been made possible by the utilization of the Tycho-1 Catalog. The ACT, combining the old Astrographic Catalog (AC) data with the Tycho-1 positions, is the best known example of this. The Tycho-2 consortium, led by E. Høg, has performed new reductions on the Tycho data. This not only has increased the astrometric and photometric accuracies of the original 1 million Tycho- 1 stars, but also has added an additional 1.5 million stars. The U.S. Naval Observatory led the effort to compute the proper motions of these 2.5 million stars. They are based not only on the AC data but also include over 140 other groundbased catalogs, all directly reduced to the Hipparcos system. The result of these efforts is the Tycho-2 Catalog, available since February 2000. Positions, proper motions, and $B_{T}$ and $V_{T}$ magnitudes are given for 2.5 million stars. The catalog is $99 \%$ complete to $\mathrm{V}=11.0$, and $90 \%$ complete to $V=11.5$. Positional accuracies at the mean epochs vary from $<10$ mas for stars $V<9$ to just under 100 mas for $V>12$. Proper motion accuracies are estimated to be $1.3 \mathrm{mas} /$ year to $3.0 \mathrm{mas} / \mathrm{year}$ for the same magnitude ranges. Photometric accuracies range from 0.02 magnitudes for the brightest stars to 0.25 magnitudes for the faintest.
\end{abstract}

\section{Introduction}

The Tycho-2 Catalog (Høg et al., 2000a; Høg et al., 2000b) is a global, reference catalog consisting of 2.5 million stars. It surpasses the ACT Reference Catalog (Urban et al., 1998) and the Tycho Reference Catalog (TRC; Kuzmin et al., 1999 ) in both size and accuracy. Compiling the catalog was a joint venture between the Copenhagen University Observatory (CUO), U.S. Naval Observatory (USNO), Astronomisches Rechen-Institut, and the European Southern Observatory. The CUO group was responsible for the re-reduction of the Tycho data and project management. The USNO staff was responsible for the compilation of the proper motions.

Tycho-2 is essentially two catalogs: 1) the re-reduced observations made with the Tycho instrument aboard the Hipparcos satellite (ESA, 1997); and 2) a reference catalog with proper motions based on other photographic and transit circle catalogs. 
Due to the new reduction of the Tycho photon counts, positions for 2.5 million stars were determined, hence the catalog has over twice the density of the original Tycho Catalog (ESA, 1997) (hereafter referred to as Tycho-1), ACT or TRC. For the proper motions, both ACT and TRC combined Tycho-1 positions with data from the Astrographic Catalog (AC) only. In Tycho-2, the proper motions are determined by utilizing a new reduction of the AC data, plus 143 other photographic and transit circle catalogs, all reduced directly to the Hipparcos system.

\section{Contents}

Astrometry For $96 \%$ of the stars, we were able to obtain proper motions. For these, the mean positions and proper motions are given for epoch J2000. Errors of each are provided. They were computed in two ways, a scatter method based on the goodness-of-fit of the catalog positions contributing to the mean position and motion, and a model method based on a priori error estimates of those catalog positions. Both are given, along with the mean epoch and number of catalog positions used.

The Tycho instrument and reductions were able to deconvolve close multiple systems with separations as small as 0.8 arcsec. For many of these pairs, only photocenter positions are available from the ground-based catalogs. The team decided that it was better to provide proper motions determined for the photocenter of the system than to not provide proper motions at all. Systems with this photocenter astrometry are flagged in the catalog.

For all stars, the observed position using only the Tycho-2 experiment is provided, along with epoch of observation and internal positional errors.

Photometry For most stars in the catalog, both the Tycho B and $\mathrm{V}\left(B_{T}, V_{T}\right)$ magnitudes are given. For every star, at least one of the values is present. Standard errors (internal) are provided.

Flags A proximity indicator, giving the distance to the nearest Tycho-2 star, is provided to aid users interested in double star work.

A flag is provided to tell users the type of processing the Tycho-2 photon counts underwent. The three types of processing were "normal", "double star", and "photocenter". In the normal star processing, no stray light from nearby stars was seen. In the double star processing, some stray light was present, but subtracted. In the photocenter processing, there was known (or suspected) stray light which could not be subtracted. Additionally, stars in common with Tycho-1 are flagged.

$C D-R O M$ The data on the CD-ROM include the main catalog (2.54 million stars), and two supplements. Supplement 1 contains 17,588 stars that are found in Hipparcos and Tycho-1, but not in Tycho-2. The reasons for exclusion from the main catalog vary. Supplement 2 contains 1146 Tycho- 1 stars that may be false or heavily disturbed by nearby, brighter stars. Note that the supplements are the only place where Hipparcos or Tycho-1 data are found.

The CD-ROM contains documentation that may be useful for users. A Guide, briefly describing the CD-ROM contents, is given. Additionally, two 
Astronomy and Astrophysics papers on the catalog's construction, verification, and characteristics are included.

\section{General Characteristics}

Some general characteristics of the Tycho-2 Catalog are presented below.

- Number of entries $=2,539,913$

- Global

- at $b=0^{\circ}$, star density is $150 \mathrm{stars} / \mathrm{deg}^{2}$

- at $b=30^{\circ}$, star density is 50 stars $/$ deg $^{2}$

- at $\mathrm{b}=90^{\circ}$, star density is $25 \mathrm{stars} / \mathrm{deg}^{2}$

- $\approx 99 \%$ complete to $V \approx 11.0$

- $\approx 90 \%$ complete to $V \approx 11.5$

- Reference catalog replacing ACT, TRC

- Observational catalog replacing Tycho-1

- Accuracies are magnitude dependent

- Most stars not in Tycho-1, ACT, and TRC are faint

- Available on CD-ROM through E. Høg or S. Urban

- Available on WWW at data centers

\section{Comparisons with Hipparcos}

Comparisons of the Tycho-2 proper motions with the Hipparcos Catalog have been investigated (Urban et al., 2000). Figure 1 shows the differences with respect to magnitude. Systematic errors appear to be under $0.3 \mathrm{mas} / \mathrm{yr}$ for most stars, with an increase toward the faint magnitudes. This increase is probably due to uncompensated magnitude equation in the photographic data used in the Tycho-2 proper motions. Readers should note that the Hipparcos Catalog is not a truly external verification of the proper motions, as it was used in the reduction of the catalogs used to compute the Tycho- 2 proper motions. 

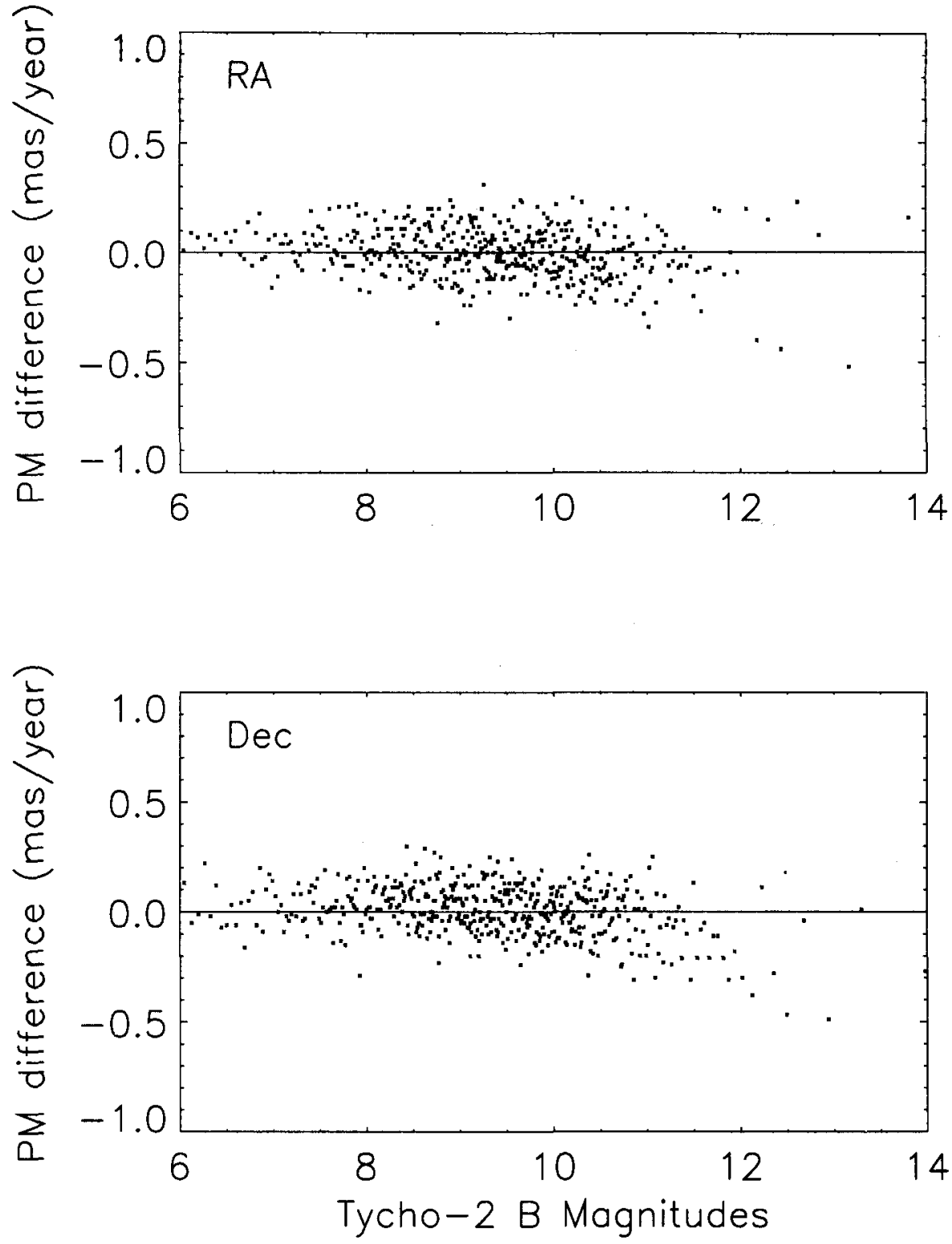

Figure 1. Differences between Tycho-2 and Hipparcos (Tycho$2-$ HIP) proper motions with respect to magnitude. Each point is a mean of 200 stars. Error bars are suppressed for clarity. 


\section{References}

ESA, 1997, SP-1200,

Høg, E., Fabricius, C., Makarov, V.V., Urban, S., Corbin, T., Wycoff, G., Bastian, U., Schwekendiek, P. \& Wicenec, A., 2000a, Astron. Astrophys., 355, L27.

Høg, E., Fabricius, C., Makarov, V.V., Bastian, U., Schwekendiek, P., Wicenec, A., Urban, S., Corbin, T. \& Wycoff, G., 2000b, Construction and Verification of the Tycho-2 Catalogue, Astron. Astrophys., submitted.

Kuzmin, A., Høg, E., Bastian, U., Fabricius, C., Kuimov, K., Lindegren, L., Makarov, V.V. \& Röser, S., 1999, Astron. Astrophys. Sup., 136, 491.

Urban, S., Corbin, T. \& Wycoff, G., 1998, Astron. J., 115, 2161.

Urban, S., Wycoff, G. \& Makarov, V.V., 2000, Comparisons of the Tycho-2 Catalogue proper motions with Hipparcos and ACT, Astron. J., in press. 